\title{
Estimación del dolor durante el perioperatorio: Una revisión narrativa de los dispositivos disponibles
}

\section{Estimative pain measurement during perioperative period: a review of current available devices}

\author{
Waldo Merino ${ }^{1, a,{ }^{*},}$ Millaray Caniuman ${ }^{1, b}$, Ignacio Muñoz ${ }^{1, b}$, Camila Oyarce $^{1, b}$ \\ Universidad de La Frontera. Temuco, Chile. \\ Magíster en epidemiología Clínica. \\ b Estudiante de Medicina.
}

Financiamiento: Sin financiamiento.

No hay conflicto de interés.

Fecha de recpción: 20 de octubre de 2020 / Fecha de aceptación: 10 de noviembre de 2021

\begin{abstract}
Pain is a complex subjective organic function which is influenced by sensorial, emotional, cognitive and behavioral elements. Despite the wide offer of pain measurement devices in the perioperative period, none of them is completely validated for their transverse use in the anesthetic practice. The aim of this review is to present the existing devices for objective pain evaluation during the perioperative period along with the scientific evidence supporting each of them. Articles from the PubMed/MEDLINE literature search engine were reviewed. As result, 37 articles were selected due to its relevance, from which 13 pain assessment devices were described, regarding its clinical relevance as well as the amount of scientific evidence found. Among them are ANI, NOL, pupillometry, qNOX, and others. The nociceptive measurement performed by most of these is based mainly on the evaluation of the autonomic nervous system activity and variations of the electroencephalographic signal. However, it is not possible to recommend any particular device. This review aims to offer a broad overview of the available options in order to estimate the role that each of them could play in clinical anesthesiology practice.
\end{abstract}

Key words: Nociceptive pain, nociception, monitoring, intraoperative, pain, postoperative.

\section{RESUMEN}

El dolor es una experiencia subjetiva compleja en la que inciden elementos sensoriales, emocionales, cognitivos y conductuales. A pesar de una amplia oferta de dispositivos para medir dolor en el perioperatorio, hoy no existe un instrumento de medición de analgesia validado y utilizado transversalmente en la práctica anestésica. El objetivo de esta revisión es presentar las actuales opciones disponibles para la medición del dolor agudo utilizadas en el período perioperatorio junto con la evidencia científica que respalda cada una de ellas. Se realizó una revisión de la literatura utilizando como fuente de búsqueda bibliográfica la base de datos MEDLINE/pubMed utilizando términos MESH. Como resultado, se seleccionaron 37 artículos de acuerdo a su importancia, a partir de los cuales se describen 13 dispositivos de valoración nociceptiva, a propósito de su relevancia clínica como también por la cantidad de evidencia científica encontrada. Entre ellos destacan ANI, NOL, pupilometría, qNOX, entre otros. La medición nociceptiva realizada por la mayoría de estos se basa principalmente en la evaluación de la actividad del sistema nervioso autónomo y variaciones de la señal electroencefalográfica. Sin embargo, no es posible recomendar algún dispositivo en particular. Esta revisión pretende ofrecer una visión amplia de las opciones disponibles con el fin de estimar el rol que cada uno de ellos podría desempeñar en la práctica clínica anestesiológica.

Palabras clave: Dolor, nocicepción, monitoreo, anestesia, término libre, perioperatorio. 


\section{Introducción}

dolor es una experiencia subjetiva compleja, en la cual se conjugan elementos sensoriales, emocionales, cognitivos y conductuales. Se considera dolor agudo como aquel que sufre un paciente quirúrgico a causa de la enfermedad previa y/o de la cirugía, el cual debe ser adecuadamente manejado durante todas las etapas relacionadas al acto quirúrgico[1].

Si bien actualmente existen métodos de monitorización de hipnosis durante la anestesia general, aún no existe un instrumento de medición de analgesia validado y utilizado transversalmente, de modo que en la práctica clínica se reduce a la evaluación de las reacciones del sistema nervioso autonómico (SNA) frente a distintos estímulos nociceptivos[2]. Sin embargo, es conocido que durante la anestesia y el acto quirúrgico, existen múltiples factores que también pueden modificar la respuesta del SNA y por ende dificultar la lectura de estos datos como parámetros de evaluación nociceptiva[3].

Por estas razones, la evaluación de la nocicepción en el período perioperatorio y trabajo de parto representa un desafío que ha impulsado la creación de diferentes herramientas cuyo propósito es objetivar la intensidad del dolor y así lograr un adecuado balance entre nocicepción-antinocicepción, lo que eventualmente podría contribuir en la reducción de las complicaciones asociadas.

El objetivo de esta revisión es presentar las actuales opciones disponibles para la medición objetiva de dolor agudo relacionadas con la práctica quirúrgica, anestesiológica y trabajo de parto junto con la evidencia científica que respalda cada una de ellas.

\section{Material y Método}

Se llevó a cabo una revisión de la literatura utilizando como fuente de búsqueda bibliográfica la base de datos MEDLINE/ pubMed. Se utilizaron términos MESH y términos libres. Términos MESH: "Nociceptive Pain", "Nociception", "Labor Pain", "Monitoring, Intraoperative", "Pain, Postoperative". Términos libres: "devices" y "perioperative". La estrategia de búsqueda realizada fue la siguiente: (((("Nociceptive Pain"[Mesh]) OR ("Nociception"[Mesh])) AND ((((("devices") OR ("perioperative")) OR ("Labor Pain"[Mesh])) OR ("Monitoring, Intraoperative"[Mesh])) OR ("Pain, Postoperative"[Mesh])))) AND ("Humans"[Mesh]). Se aplicaron filtros por idioma inglés y para humanos en los últimos diez años, resultando un total de 116 artículos. La búsqueda fue realizada por cada uno de los autores, y según un tamizaje por título y resumen, se escogieron un total de 30 publicaciones, las cuales fueron distribuidas y cruzadas, siendo seleccionadas aquellas que contaron con dos o más concordancias, resultando un total de 21 artículos. Además, se añadieron 16 trabajos conocidos previamente por los autores o rescatados de la bibliografías de los papers seleccionados de la búsqueda, alcanzando un total de 37 artículos.

\section{Resultados}

En consecuencia de los 37 artículos seleccionados, se describen a continuación 13 dispositivos de valoración nociceptiva, a propósito de su relevancia clínica como también de la cantidad de evidencia científica actualizada.

\section{1. Índice de nocicepción de analgesia (ANI)}

Es un instrumento simple, no invasivo, que provee un índice numérico adimensional entre 0-100, que revela el balance entre el proceso neuronal mediante el cual, se codifican y procesan estímulos potencialmente dañinos y, analgesia durante la anestesia general. ANI se basa en el análisis continuo de la variabilidad de la frecuencia cardiaca obtenida desde un electrocardiograma (ECG) estándar, que refleja variabilidad del tono parasimpático, a través de su promedio durante los últimos 60 segundos[4]. Esto lo realiza mediante el uso de información proveniente de 2 electrodos ubicados en el esternón y en la línea medio axilar[5].

El estrés quirúrgico lleva al predominio del SNA simpático y consecuentemente a una disminución del índice ANI. Estudios señalan que los puntajes $<50$ sugieren presencia de estímulos nociceptivos, inducidos experimentalmente a través de electricidad o intraoperatoriamente con incisión quirúrgica[6].

Es importante tener en cuenta que diversos factores pueden alterar la veracidad de los puntajes ANI, tales como bradipnea o apnea[7], ya que según Logier $\mathrm{R}$ y cols., un estímulo doloroso es capaz de generar variaciones en el patrón de arritmia sinusal respiratoria. Otras variables a considerar, para la correcta interpretación del ANI son los cambios en la hipnosis, ritmo cardíaco sinusal no regular, marcapasos implantados, agentes antimuscarínicos, agonistas $\alpha 2$ adrenérgicos, antagonistas $\beta 1$ adrenérgicos y antiarrítmicos[2],[8].

Según un estudio realizado por Dundar y cols., acerca del monitoreo intraoperatorio mediante $\mathrm{ANI}$, se evidenció que su uso optimiza significativamente el consumo de analgésicos, debido a que evidencia variaciones hemodinámicas asociadas a estímulos nocivos[9]. En concordancia con estos resultados, el estudio realizado por Upton y cols., demuestra también la utilidad del ANI para administrar dosis de fentanilo de manera más equilibrada en pacientes bajo anestesia general, disminuyendo así los niveles de dolor posoperatorio. Sin embargo, los mismos autores observaron que la relación entre ANI y el requerimiento de analgesia puede no ser lineal para valores extremos de ANI, siendo necesaria más investigación en torno a la dosis ideal de analgesia para estos casos[8].

Por último, de acuerdo a Ledowski y cols., como también según Kaushic y cols., quienes estudiaron la eficacia de ANI para evaluar nocicepción durante el posoperatorio, éste no mostró evidencia significativa para considerarse una herramienta confiable que refleje el dolor agudo durante este período, cuando es comparado con una escala de calificación numérica[10],[11], mientras que Boselli y cols., concluyen que ANI representa una buena alternativa para evaluar la ausencia de dolor posoperatorio inmediato[12].

\section{Ecuación de respuesta nociceptiva (NR)}

Ecuación matemática que evalúa niveles nociceptivos de pacientes bajo anestesia general, utilizando parámetros hemodinámicos de frecuencia cardíaca, presión arterial sistólica e índice de perfusión, cuya fórmula matemática es: NR=-1+2/ $(1+\exp (-0.01 \mathrm{HR}-0.02 \mathrm{SBP}+0.17 \mathrm{PI}))$. A través de una cohorte retrospectiva, Miyawaki y cols., evaluaron las diferencias en los niveles nociceptivos de pacientes sometidos a una videotora- 
coscopía, comparando los resultados de la ecuación según si recibieron anestesia general o anestesia general más bloqueo paravertebral. Sus resultados mostraron niveles de nocicepción significativamente más altos en los pacientes que no recibieron el bloqueo paravertebral, razón por la cual concluyeron que la ecuación de respuesta nociceptiva, sería un método disponible para la medición objetiva del dolor[13].

\section{Pupilometría}

Herramienta no invasiva que mide el reflejo de dilatación pupilar (RDP), que a través de la grabación de un video infrarrojo valora cuantitativamente el tamaño de la pupila. Es un mecanismo bien tolerado que aporta información relacionada a dos vías de procesamiento nociceptivo: la reactividad autónoma relacionada al dolor, y el componente ascendente del sistema somatosensorial. Wildemeersch y cols., utilizaron un protocolo de medición del índice de dolor pupilar (PPI), en pacientes bajo ventilación mecánica, observando que es capaz de entregar información relacionada al nivel de analgesia del paciente[14]. Este protocolo ha sido previamente utilizado en adultos anestesiados, denotando una correlación significativa entre la PDR y la administración de opioides[15]. Esto concuerda con Sabourdin y cols., quienes demostraron que la PDR puede ser empleada para guiar la administración intraoperatoria de remifentanilo, reduciendo así el consumo intra y posoperatorio de opioides[16].

\section{Reflejo de flexión nociceptivo (RFN)}

Método no invasivo que valora el reflejo de flexión nociceptivo, que evalúa la actividad de las fibras aferentes primarias en la transmisión de señales nociceptivas, desde los nociceptores periféricos, a la cadena simpática. Según Rhudy y cols., existe una respuesta tardía del RFN medida a través de electromiografía (EMG) sobre el bíceps femoral, después de la activación del nociceptor[17]. Wildemeersch y cols., observaron que mediante estimulaciones eléctricas crecientes con electrodos cutáneos en el maléolo lateral se puede evaluar la respuesta refleja, considerando tiempo y amplitud a través de la EMG, lo que se asocia a los niveles de nocicepción del paciente, demostrando que la NFR puede utilizarse rutinariamente en pacientes críticos[14].

\section{5. Índice pletismográfico (SPI)}

Dispositivo que evalúa el tono simpático, mediante la medición combinada del intervalo normalizado entre latidos cardíacos (HBI norm) y la amplitud de pulso pletismográfico normalizado (PPGA norm), según la fórmula[18]: SPI= $100-$ 0,7 PPGAnorm +0,3 HBI norm. Se calcula utilizando un sensor fotopletismográfico que mide cambios de volumen en el lecho microvascular de los tejidos. Sus valores oscilan entre 0 y 100 (bajo y alto nivel de estrés respectivamente). Se ha demostrado que un valor SPI intraoperatorio entre 20 y 50 reduce el consumo de remifentanilo y mejora la estabilidad hemodinámica[18].

Para efectos de esta revisión se reconocen dos:

1. SPI, GE Healthcare, Chicago, IL, EE. UU.

2. GE Healthcare, Helsinki, Finlandia; originalmente Ilamado "Índice de estrés quirúrgico" [SSI].

- Software de Monitoreo Basado en Evidencia (EBMi)

EBMi Custos $^{\odot}$, dispositivo basado en SPI, tiene como objetivo advertir la aparición de variaciones patológicas durante el periodo de anestesia[19]. A diferencia de otros instrumentos de monitoreo, EBMi no se limita a alertar cuando los valores sobrepasan un umbral, sino que recopila datos periodicamente de estos monitores clásicos, evaluando cambios paramétricos a lo largo del tiempo, sugieriendo un diagnóstico[18].

La alerta de "respuesta simpática" corresponde a la conjunción de una tendencia ascendente de la frecuencia cardíaca y presión arterial, y una tendencia descendente en la amplitud de la onda de pulso pletismográfica[18].

Defresne y cols., analizaron datos recopilados de un estudio publicado anteriormente de pacientes sometidos a anestesia general, donde evaluaron la aparición de cambios relevantes y sostenidos en el SPI (superior a 60 durante al menos 20 segundos), para determinar si esos episodios habrían generado alertas de EBMi y si fueron precedidos por cambios fisiológicos indicativos de un tono simpático aumentado[20]. Ellos observaron un número menor de alertas EBMi versus episodios SPI sobre 60. Sin embargo, EBMi además advirtió cambios atípicos como aumentos en la presión arterial sin taquicardia o disminuciones aisladas de la amplitud del pulso pletismográfico. Además, los autores destacaron que al ocurrir una cantidad importante de alertas EBMi, cuando el SPI era menor de 60, sugiere mayor especificidad que el SPI. Sin embargo, concluyen que son necesarios estudios prospectivos aleatorizados para una mejor comparación entre estos[18].

\section{Electroencefalograma (EEG)}

Existen métodos derivados de la señal del EEG utilizados como base para el diseño de indicadores que evalúan el efecto hipnótico, como el análisis biespectral (BIS) de la señal de EEG, análisis de entropía y potenciales evocados auditivos, entre otros. Sin embargo, en cuanto a la medición analgésica, no se ha logrado desarrollar un sistema completamente certero[21].

A continuación se describen alternativas derivadas de la señal electroencefalográfica para medir dolor.

- Análisis espectral del EEG

Melia y cols., evaluaron la utilidad de las mediciones extraídas de EEG para evaluar las respuestas al dolor durante la sedación. Los resultados indicaron que las mediciones lineales y no lineales del EEG frontal, filtradas en altas frecuencias, que contenían también componentes electromiográficos, son una metodología prometedora para la predicción no invasiva de las respuestas al dolor[22].

Estudios realizados por Okolo y cols., en pacientes despiertos no sometidos a analgesia, se analizó la utilidad del EEG con electrodos sin gel, asociado a una máquina de soporte de vectores para predecir cambios en tiempo real, en la nocicepción ante un estímulo único creciente de dolor. Las ondas del EEG permiten obtener un espectro de variaciones frecuenciales de las señales de onda completa, útiles en el seguimiento de la densidad espectral para caracterizar la actividad cerebral ante estímulos dolorosos, mostrando cambios significativos en todas las potencias de las bandas de frecuencia (Aumento del delta de 1 a $4 \mathrm{~Hz}$, beta de 18 a $32 \mathrm{~Hz}$, gamma de 32 a $72 \mathrm{~Hz}$ y disminuciones en theta 4-8 $\mathrm{Hz}$, alfa1 en 8-10Hz, alpha 2 en 10-12 Hz)[23].

- Entropía de Datex Ohmeda S/5 (EDOS/5) EDOS/5, elaborado por División Datex-Ohmeda, Instrumentarium Corp., Helsinki, Finlandia, es un dispositivo basado 
en la entropía que consta de un sensor de tres electrodos ubicados en la frente, capaz de capturar un electroencefalograma frontal de una derivación[24].

Este monitoriza la irregularidad de señales del EEG a través de dos parámetros: Entropía de estado (SE), la cual registra ondas eléctricas cerebrales, en el rango de $0,8 \mathrm{~Hz}$ a $32 \mathrm{~Hz}$, y entropía de potencia (RE), que incluye el registro del EEG más la actividad muscular, utilizando frecuencias de hasta $47 \mathrm{~Hz}[24]$.

Guerrero y cols., estudiaron la relación entre la electromiografía frontal (EMGf) y la nocicepción a concentraciones anestésicas donde el movimiento no interfiere con EMGf, comparando tres indicadores de EEG durante anestesia profunda; RE, SE Y BIS (índice biespectral). Ellos observaron que en pacientes bajo anestesia general utilizando sevoflurano, el RE es el único parámetro capaz de detectar variaciones después de la estimulación nociceptiva[25].

- qNOX y qCON. Debido a que el electroencefalograma es una medida directa del nivel de actividad cerebral, éste junto a señales electromiográficas pueden cuantificar la hipnosis y niveles nociceptivos[3]. El índice qCON, mediante el Monitor qCON 2000; Quantium Medical, España, evalúa el efecto hipnótico y el índice qNOX la nocicepción. Ambos se basan en la combinación de bandas de frecuencia conectadas a un Sistema de Inferencia Neurodifusa Adaptativa que genera una escala de 0 a 99, donde un qNOX $<40$ significa una probabilidad muy baja, entre 40 y 60 una probabilidad baja y valores $>60$ una alta probabilidad de respuesta a estímulos nocivos[3],[26].

Melia y cols., evaluaron su utilidad en diversos procedimientos quirúrgicos, encontrando que qCON fue capaz de predecir la pérdida de conciencia, mientras que qNOX pudo predecir la respuesta ante una estimulación nociva, como la inserción de la máscara laringea. Ambos índices detectaron diferencias entre los tiempos de acción de los agentes hipnóticos y analgésicos: qCON mostró una disminución más rápida durante la inducción, mientras que qNOX mostró un aumento más rápido durante la recuperación. A partir de esto, los autores suponen que la probabilidad de respuesta a estímulos nocivos evaluada por el qNOX, disminuye después de la pérdida de conciencia y aumenta antes de que el paciente la recupere, según la evaluación de qCON. Por tanto, el qNOX podría interpretarse como un índice de excitación, lo que indica que es probable que el paciente se despierte. Estos resultados confirman que el uso conjunto de estos índices mejoran la detección del efecto de los analgésicos que se inducen en el EEG[22].

De manera similar, Jensen y cols., evaluando la efectividad de ambos índices en pacientes bajo anestesia general, obtuvieron que tanto qCON como qNOX pudieron detectar movimiento como respuesta a la estimulación nociva, y señalan que probablemente no sea posible utilizarlos por separado, ya que cuando un paciente está bajo la influencia de un fuerte efecto hipnótico, por ejemplo un qCON de 20, entonces la probabilidad de respuesta a estímulos nocivos es menor[3].

En general, qNOX representa una buena alternativa para evaluar la nocicepción, ya que no depende de la actividad autonómica periférica. Esto permite una puntuación más sólida frente a la influencia de medicamentos con efecto cardiovascular y comorbilidades del paciente. Sin embargo, los creadores advierten de la posibilidad que agentes bloqueadores neuromusculares alteren la puntuación debido al uso de señales electromiográficas[26].

\section{Espectroscopía infrarroja cercana (NIRS)}

Método no invasivo de monitorización de la corteza cerebral que utiliza un espectrómetro infrarrojo que opera a longitudes de onda entre 690 y 830 Hz (TechEn Inc. MA, EE.UU., Sistema CW7). Detecta cambios en las concentraciones de hemoglobina oxigenada y desoxigenada, los que reflejan variaciones en el volumen sanguíneo total. Las fluctuaciones hemodinámicas son resultado de la vasodilatación cerebral producida con el fin de aumentar el flujo sanguíneo a áreas cerebrales de mayor actividad[27]. El dolor involucra regiones cerebrales observables mediante la espectroscopía infrarroja, como el lóbulo frontal y el área somatosensorial, por lo que a priori, podría utilizarse como una herramienta para la medición objetiva del dolor[27].

Aasted y cols., realizaron pruebas con estímulos nociceptivos de diferente intensidad para determinar regiones cerebrales específicas de activación en sujetos sanos despiertos. Se aplicaron 12 estímulos de baja intensidad (3/10 en la escala numérica del dolor) y 12 estímulos de alta intensidad (7/10 en la misma escala) de forma secuencial. Sus resultados muestran que estímulos nociceptivos generan cambios hemodinámicos significativos en el lóbulo frontal proporcionales la intensidad del estímulo aplicado[28]. De manera similar, Becerra y cols., en pacientes sometidos a colonoscopías, encontraron una asociación entre los estímulos nociceptivos del procedimiento y la activación de la corteza frontal[27].

\section{Conductancia de la piel (SC)}

Dado que las glándulas sudoríparas de las palmas de manos y pies reciben inervación exclusiva de fibras nerviosas simpáticas, una descarga de este sistema desencadenará un aumento en la actividad de dichas glándulas, produciendo un flujo de sudor en la superficie de la piel, provocando así un aumento de la conductancia de la piel medible a través de un dispositivo no invasivo: Stress Detector TM; MedStorm, Oslo, Noruega. Se entiende entonces que, variaciones de la conductancia cutánea son un reflejo de la activación del SNA simpático periférico. Es un dispositivo no invasivo que consta de tres electrodos ubicados en la mano o pie del paciente. La SC mostró una buena correlación con la evaluación del dolor en adultos despiertos[29] y niños[30]. También se estudió en adultos anestesiados, durante la inducción y el despertar, con resultados concordantes[31]. Sabourdin y cols., estudiaron su eficacia en la evaluación de la nocicepción en niños sometidos a anestesia general, y a su vez la compararon con el ANI. Concluyeron que si bien el valor SC no se ve influenciado por fluctuaciones hemodinámicas, relajantes musculares, ventilación o temperatura (excepto en condiciones extremas), el ANI proveería una evaluación nociceptiva más sensible que la SC[32].

\section{9. Índice de nivel de nocicepción (NOL)}

Instrumento multiparamétrico que entrega un número adimensional entre 0 y 100 (números menores indican menos nocicepción), basado en el análisis algorítmico de cuatro parámetros: fotopletismografía, respuesta galvánica de la piel, temperatura y acelerometría. Todos los sensores para la medición 
de los parámetros están integrados en un dispositivo digital, similar a un oxímetro de pulso. Esta herramienta fue puesta a prueba por Stockle y cols., a través de la aplicación de estímulos dolorosos estandarizados, en 40 pacientes bajo anestesia general, que fueron medidos a través de NOL luego de la administración de varias y sucesivas dosis de remifentanilo. Este estudió evidenció que NOL es capaz de discriminar estímulos nociceptivos en pacientes bajo anestesia general, con diferencias estadísticamente significativas entre las dosis de remifentanilo, es decir, cuanto mayor es el remifentanilo, menor es la respuesta NOL[33].

Por otra parte, Meijer y cols., compararon niveles de dolor posoperatorio en 50 pacientes bajo anestesia general sometidos a cirugía abdominal que se dividieron aleatoriamente en dos grupos, según si recibieron anestesia guiada por NOL o por parámetros hemodinámicos estándar. Sus resultados no evidenciaron diferencias significativas relacionadas al consumo de opioides. Sin embargo, encontraron diferencias significativas en los niveles de dolor posoperatorio medidos a través de la escala NRS. Además, un hallazgo importante fue que los sujetos con régimen anestésico administrado bajo NOL, registraron una concentración de hormonas relacionadas al estrés en $50 \%$ menos, durante y posteriormente al acto quirúrgico[34].

Por su parte, Bollag y cols., evaluaron los cambios producidos en los puntajes de NOL luego de una incisión para trocar de videotoracoscopía en 16 pacientes con (8) y sin (8) anestesia epidural torácica. Sus resultados evidenciaron una diferencia estadísticamente significativa entre ambos grupos, con puntajes NOL mayores en aquellos que no recibieron anestesia epidural. Secundariamente, al comparar el índice NOL con la frecuencia cardíaca como indicadores de nocicepción, encontraron que el primero aumentó en $90 \%$ después de la intubación, mientras el segundo aumentó solo en $12 \%$ para el mismo estímulo. Este hecho podría preliminarmente asociarse a una mejor sensibilidad del dispositivo para detectar estímulos nocivos, como la intubación[35].

\section{CARDEAN}

El barorreflejo cardíaco, es un mecanismo de regulación de la presión arterial (PA) que actúa fisiológicamente mediado por el SNA ante cambios agudos de ésta, de tal manera que al ascender bruscamente la PA, se estiran los mecanorreceptores aumentando la frecuencia de descargas en el núcleo del tracto solitario, produciendo una disminución del inotropismo, además de vasodilatación y bradicardia, a través de neuronas inhibidoras del SNA simpático y excitatorias del parasimpático. En respuesta a descensos bruscos de PA, los barorreceptores de alta presión disminuyen sus descargas, provocando una respuesta simpática de taquicardia, aumento del inotropismo cardíaco, vasoconstricción y normalización de la PA.

Ante un estímulo quirúrgico, el barorreflejo cardiaco se transforma de modo que la respuesta ante hipertensión pasa a ser taquicardia, mediante un patrón Ilamado: reflejo somatosimpático[36].

CARDEANTM 2.0 (dispositivo creado por Alpha-2 Ltda, Lyon, Francia) monitorea en línea el latido a latido de cada paciente, calculando un índice cardiovascular que mide profundidad analgésica, mediante la detección del efecto de estímulos nociceptivos, reflejado en latidos de menor amplitud y menor duración (10-20s). Utiliza una escala de 0-100, que describe un patrón de hipertensión asociada a taquicardia durante el proceso, traduciéndose en un mayor valor CARDEAN, mientras mayor sea la taquicardia.

Rossi y cols., utilizaron el CARDEAN como mecanismo para identificar antinocicepción intraoperatoria inadecuada, así como también durante la inducción con opioides, demostrando que éste es capaz de rastrear despertares microsimpáticos antecediendo la aparición de signos clínicos como taquicardia, hipertensión arterial, movimiento y contracciones musculares[36]. Cividjian y cols., estudiando pacientes bajo anestesia general, confirmaron a CARDEAN como una guía confiable para la administración de opioides, siendo capaz de reducir en $51 \%$ el número de movimientos en la colonoscopia de forma espontánea[37]. Sin embargo, el dispositivo presenta un determinado retraso, en aquellos casos en que los signos clínicamente observables de una inadecuada antinocicepción y que ocurren súbitamente, no son precedidos por los despertares microsimpáticos. En estos casos el retraso resulta en una predicción inadecuada[37].

\section{Discusión}

Como resultado preliminar de la revisión, se hallaron más de 20 dispositivos de valoración nociceptiva, de los cuales fueron seleccionados 13 para una descripción más detallada, a propósito de su relevancia clínica, como también de la cantidad de evidencia científica actualizada.

ANI es el dispositivo que más ha sido investigado, destacándose por ser una herramienta monoparamétrica, sencilla de utilizar y capaz de optimizar significativamente el uso de analgésicos intraoperatorios, lo que supone un beneficio clínico relevante. Sin embargo, su capacidad para valorar el dolor posoperatorio permanece en discordancia entre los autores. En la misma línea, tanto SPI como pupilometría, han demostrado ser útiles en la reducción del consumo de opioides intraoperatorios, sin embargo, estos dispositivos han sido menos investigados, por lo que su validación clínica aún no es concluyente.

Por otro lado, dentro de los instrumentos basados en el EEG, existen variadas apreciaciones entorno a su rendimiento dependiendo de los parámetros utilizados para realizar la medición objetiva del dolor. Entre ellos, el índice qCON junto al índice qNOX empleados en conjunto, mejoran la detección del efecto de los analgésicos que se producen en el EEG. Además, qNOX surge como una buena alternativa a la mayoría de los dispositivos ya que no depende de la actividad nerviosa autonómica. Sin embargo, agentes bloqueadores neuromusculares podrían distorsionar la puntuación debido al uso de señales electromiográficas. Otro de los instrumentos derivado del EEG, es la entropía, la cual como monitor objetivo del dolor resulta prometedor al ser capaz de identificar la irregularidad de las señales propiciadas por el EEG.

Finalmente, NOL surge como una herramienta que destaca por realizar una medición multiparamétrica, de fácil utilización y por ser capaz de discriminar estímulos nociceptivos con diferencias estadísticamente significativas entre variadas dosis de remifentanilo. Pese a lo prometedor que parece ser este dispositivo, es necesaria la realización de más estudios para confirmar su verdadera utilidad en la práctica clínica.

Nos parece relevante destacar que no se encontró evidencia 
relacionada a la medición del dolor durante el trabajo de parto, por lo que la estimación de la intensidad del dolor aún depende de la apreciación subjetiva de la paciente.

Si bien, queda a la vista que existen diversas herramientas que pretenden medir el dolor de manera objetiva, ninguna de ellas lo logra con total precisión por lo que, como concluyen la mayoría de los autores citados, se hace necesario continuar investigando para complementar la evidencia existente actualmente, con el fin de mejorarlas y validarlas.

Dadas las razones mencionadas anteriormente, no es posible recomendar algún dispositivo en particular. Sin embargo, se espera que con esta revisión el lector obtenga una visión amplia de las opciones disponibles en el mercado y pueda reconocer las principales características que presentan los equipos, con el fin de estimar el rol que cada uno de ellos podría desempeñar en su práctica clínica anestesiológica.

\section{Referencias}

1. Stowell JR, Kessler R, Lewiss RE, Barjaktarevic I, Bhattarai B, Ayutyanont $\mathrm{N}$, et al. Critical care ultrasound: A national survey across specialties. J Clin Ultrasound. 2018 Mar;46(3):167-77. https://doi.org/10.1002/jcu.22559 PMID:29131347

2. Whitson MR, Mayo PH. Ultrasonography in the emergency department. Crit Care. 2016 Aug;20(1):227. https://doi. org/10.1186/s13054-016-1399-x PMID:27523885

3. Charron C, Repessé X, Bodson L, Au SM, Vieillard-Baron A. Ten good reasons why everybody can and should perform cardiac ultrasound in the ICU. Anaesthesiol Intensive Ther. 2014 Nov-Dec;46(5):319-22. https://doi.org/10.5603/AIT.2014.0055 PMID:25432551

4. Yin W, Li Y, Zeng X, Qin Y, Wang D, Zou T, et al. The utilization of critical care ultrasound to assess hemodynamics and lung pathology on ICU admission and the potential for predicting outcome. PLoS One. 2017 Aug;12(8):e0182881. https://doi. org/10.1371/journal.pone.0182881 PMID:28806783

5. Zieleskiewicz L, Bouvet L, Einav S, Duclos G, Leone M. Diagnostic point-of-care ultrasound: applications in obstetric anaesthetic management. Anaesthesia. 2018 Oct;73(10):1265-79. https:// doi.org/10.1111/anae.14354 PMID:30047997

6. AlEassa EM, Ziesmann MT, Kirkpatrick AW, Wurster CL, Gillman LM. Point of care ultrasonography use and training among trauma providers across Canada. Can J Surg. 2016 Feb;59(1):6-8. https://doi.org/10.1503/cjs.010215 PMID:26574705

7. Enriquez JL, Wu TS. An introduction to ultrasound equipment and knobology. Crit Care Clin. 2014 Jan;30(1):25-45. https://doi. org/10.1016/j.ccc.2013.08.006 PMID:24295840

8. Estenssoro E, Alegría L, Murias G, Friedman G, Castro R, Nin Vaeza N, et al.; Latin-American Intensive Care Network (LIVEN). Organizational Issues, Structure, and Processes of Care in 257 ICUs in Latin America: A Study From the Latin America Intensive Care Network. Crit Care Med. 2017 Aug;45(8):1325-36. https:// doi.org/10.1097/CCM.0000000000002413 PMID:28437376

9. Blanco P. Critical Care Ultrasound in Developing Countries of Latin America: A Long Way to Go. J Ultrasound Med. 2019 Jun;38(6):1617-8. https://doi.org/10.1002/jum.14833 PMID:30269337

10. Galarza L, Wong A, Malbrain ML. The state of critical care ultrasound training in Europe: A survey of trainers and a comparison of available accreditation programmes. Anaesthesiol Intensive Ther. 2017;49(5):382-6. https://doi.org/10.5603/AIT.a2017.0075 PMID:29192421

11. Mourtzakis M, Parry S, Connolly B, Puthucheary Z. Skeletal muscle ultrasound in critical care: A tool in need of translation. Ann Am Thorac Soc. 2017 Oct;14(10):1495-503. https://doi. org/10.1513/AnnalsATS.201612-967PS PMID:28820608

12. Robba C, Cardim D, Tajsic T, Pietersen J, Bulman M, Donnelly J, et al. Ultrasound non-invasive measurement of intracranial pressure in neurointensive care: A prospective observational study. PLoS Med. 2017 Jul;14(7):e1002356. https://doi.org/10.1371/ journal.pmed.1002356 PMID:28742869

13. Cardim D, Robba C, Bohdanowicz M, Donnelly J, Cabella B, Liu $X$, et al. Non-invasive Monitoring of Intracranial Pressure Using Transcranial Doppler Ultrasonography: Is It Possible? Neurocrit Care. 2016 Dec;25(3):473-91. https://doi.org/10.1007/s12028016-0258-6 PMID:26940914

14. Vieillard-Baron A, Mayo P. Lung ultrasonography and echocardiography in the Intensive Care Unit: a combined and practical approach. Minerva Anestesiol. 2018 Mar;84(3):398-408. https:// doi.org/10.23736/S0375-9393.17.12264-9 PMID:29027776

15. Antonio AC, Knorst MM, Teixeira C. Lung ultrasound prior to spontaneous breathing trial is not helpful in the decision to wean. Respir Care. 2018 Jul;63(7):873-8. https://doi. org/10.4187/respcare.05817 PMID:29666295

16. Brogi E, Gargani L, Bignami E, Barbariol F, Marra A, Forfori F, et al. Thoracic ultrasound for pleural effusion in the intensive care unit: a narrative review from diagnosis to treatment. Crit Care. 2017;21(1):1-11. https://doi.org/10.1186/s13054-017-1897-5 PMID:28057037

17. See KC, Ong V, Tan YL, Sahagun J, Taculod J. Chest radiography versus lung ultrasound for identification of acute respiratory distress syndrome: a retrospective observational study. Crit Care. 2018 Aug;22(1):203. https://doi.org/10.1186/s13054-018-2105-y PMID:30119687

18. Umbrello M, Formenti P. Ultrasonographic Assessment of Diaphragm Function in Critically III Subjects. Respir Care. 2016 Apr;61(4):542-55. https://doi.org/10.4187/respcare.04412 PMID:26814218

19. Jiménez Restrepo JN, León OJ, Quevedo Florez LA. Ocular Ultrasonography: A Useful Instrument in Patients with Trauma Brain Injury in Emergency Service. Emerg Med Int. 2019 Jan;2019:9215853. https://doi.org/10.1155/2019/9215853 PMID:30805217

20. Camacho JC, Coursey-moreno C, Telleria JC, Aguirre DA, Torres WE, Mittal PK. Nonvascular Post - Liver Trans plantation Complications : From US Screening to Cross-sectional and. Radiographics. 2015;1963(2):87-104. https://doi.org/10.1148/rg.351130023 PMID:25590390

21. Caballero AF, Villarreal K. Ultrasound for central vascular access. A safety concept that is renewed day by day [Review]. Colomb J Anesthesiol. 2018;46:35-41. https://doi.org/10.1097/ CJ9.0000000000000043.

22. Sippel S, Muruganandan K, Levine A, Shah S. Review article: use of ultrasound in the developing world. Int J Emerg Med. 2011 Dec;4(1):72. https://doi.org/10.1186/1865-1380-4-72 PMID:22152055

23. Blaivas M, Kuhn W, Reynolds B, Brannam L. Change in differential diagnosis and patient management with the use of portable ultrasound in a remote setting. Wilderness Environ Med. 
2005;16(1):38-41. https://doi.org/10.1580/1080-6032(2005)16[ 38:CIDDAP]2.0.CO;2 PMID:15813146

24. Panés J, Bouzas R, Chaparro M, García-Sánchez V, Gisbert JP, Martínez de Guereñu B, et al. Systematic review: the use of ultrasonography, computed tomography and magnetic resonance imaging for the diagnosis, assessment of activity and abdominal complications of Crohn's disease. Aliment Pharmacol Ther. 2011 Jul;34(2):125-45. https://doi.org/10.1111/j.13652036.2011.04710.x PMID:21615440

25. Jain R, Jain N, Sheikh T, Yadav C. Early scaphoid fractures are better diagnosed with ultrasonography than $\mathrm{X}$-rays: $\mathrm{A}$ prospective study over 114 patients. Chin J Traumatol. 2018 Aug;21(4):206-10. https://doi.org/10.1016/j.cjtee.2017.09.004 PMID:29551580

26. Marhofer $\mathrm{P}$, Frickey N. Ultrasonographic guidance in pediatric regional anesthesia Part 1: theoretical background. Paediatr Anaesth. 2006 Oct;16(10):1008-18. https://doi.org/10.1111/j.14609592.2006.02020.x PMID:16972828

27. Andruszkiewicz P, Sobczyk D. Ultrasound in critical care. Anaesthesiol Intensive Ther. 2013 Jul-Sep;45(3):177-81. https://doi. org/10.5603/AIT.2013.0036 PMID:24092516

28. Mok D, Schwarz SK, Rondi K. Point-of-care ultrasonography in Canadian anesthesiology residency programs: a national survey of program directors. Can J Anaesth. 2017 Oct;64(10):1023-36. https://doi.org/10.1007/s12630-017-0935-8 PMID:28755100

29. Atkinson P, Ross P, Henneberry R. Coming of age: emergency point of care ultrasonography in Canada. CJEM. 2014 Jul;16(4):265-8. https://doi.org/10.2310/8000.2013.131186 PMID:25060078

30. Ma IW, Steinmetz P, Weerdenburg K, Woo MY, Olszynski P, Heslop CL, et al. The Canadian Medical Student Ultrasound Curriculum: A Statement From the Canadian Ultrasound Consensus for Undergraduate Medical Education Group. J Ultrasound Med. 2020 Jul;39(7):1279-87. https://doi.org/10.1002/jum.15218 PMID:31943311

31. Cheung JC, Ho LT, Cheng JV, Cham EY, Lam KN. Staff safety during emergency airway management for COVID-19 in Hong Kong. Lancet Respir Med. 2020 Apr;8(4):e19. https://doi. org/10.1016/S2213-2600(20)30084-9 PMID:32105633

32. Buonsenso D, Pata D, Chiaretti A. COVID-19 outbreak: less stethoscope, more ultrasound. Lancet Respir Med. 2020 May;8(5):e27. https://doi.org/10.1016/\$2213-2600(20)30120-X PMID:32203708

33. Trivedi JN. An economical model for mastering the art of intubation with different video laryngoscopes. Indian J Anaesth. 2014 Jul;58(4):394-6. https://doi.org/10.4103/0019-5049.138967 PMID:25197105

34. Soldati G, Smargiassi A, Inchingolo R, Buonsenso D, Perrone $T$, Briganti DF, et al. Is There a Role for Lung Ultrasound During the COVID-19 Pandemic? J Ultrasound Med. 2020 Jul;39(7):1459-62. https://doi.org/10.1002/jum.15284 PMID:32198775

35. Buonsenso D, Piano A, Raffaelli F, Bonadia N, de Gaetano Donati $\mathrm{K}$, Franceschi F. Point-of-Care Lung Ultrasound findings in novel coronavirus disease-19 pnemoniae: a case report and potential applications during COVID-19 outbreak. Eur Rev Med Pharmacol Sci. 2020 Mar;24(5):2776-80. https://doi.org/10.26355/eurrev_202003_20549 PMID:32196627

36. Safaee Fakhr B, Araujo Morais CC, De Santis Santiago RR, Di Fenza R, Gibson LE, Restrepo PA, et al. Bedside monitoring of lung perfusion by electrical impedance tomography in the time of COVID-19. Br J Anaesth. 2020 Nov;125(5):e434-6. https://doi. org/10.1016/j.bja.2020.08.001 PMID:32859359

37. Moerer O, Hahn G, Quintel M. Lung impedance measurements to monitor alveolar ventilation. Curr Opin Crit Care. 2011 Jun;17(3):260-7. https://doi.org/10.1097/MCC. Ob013e3283463c9c PMID:21478747

38. Liu S, Tan L, Möller K, Frerichs I, Yu T, Liu L, et al. Identification of regional overdistension, recruitment and cyclic alveolar collapse with electrical impedance tomography in an experimental ARDS model. Crit Care. 2016 May;20(1):119. https://doi.org/10.1186/ s13054-016-1300-y PMID:27142073 